\title{
Role of high-mobility group box I in patients with acute obstructive suppurative cholangitis-induced sepsis
}

\author{
Akanand Singh' \\ Yi Feng' \\ Nisha Mahato² \\ Jinzheng $\mathrm{Li}^{\prime}$ \\ Chuanxin $\mathrm{Wu}^{\prime}$ \\ Jianping Gong' \\ 'Chongqing Key Laboratory \\ of Hepatobiliary Surgery and \\ Department of Hepatobiliary \\ Surgery, The Second Affiliated \\ Hospital of Chongqing Medical \\ University, Chongqing, People's \\ Republic of China; ${ }^{2}$ Department of \\ Prosthodontics, The Affiliated Hospital \\ of Stomatology, Chongqing Medical \\ University, Chongqing, People's \\ Republic of China
}

This article was published in the following Dove Press journal:

Journal of Inflammation Research

II March 2015

Number of times this article has been viewed

Background: High-mobility group box 1 (HMGB1) is a proinflammatory cytokine that plays an active role during the pathogenesis of inflammatory processes. The primary aim of this study was to detect whether HMGB1 is involved in the pathogenesis of acute obstructive suppurative cholangitis (AOSC).

Methods: We collected peripheral blood samples from 23 patients with AOSC and 23 healthy volunteers who served as normal controls. All participants were tested for HMGB1 mRNA level, HMGB1 protein, tumor necrosis factor alpha (TNF-alpha), and interleukin 10 (IL-10). HMGB1 mRNA levels were tested using real-time polymerase chain reaction. HMGB1 protein expression was measured using Western blot. TNF-alpha and IL-10 were tested using enzymelinked immunosorbent assay.

Results: The expression of HMGB1 mRNA and HMGB1 protein was higher in the AOSC group than in the normal controls $(P<0.01)$, and the levels gradually decreased to normal after treatment of the disease $(P<0.01)$. The content of TNF-alpha and IL-10 in peripheral blood of patients with AOSC was significantly higher than that of normal controls $(P<0.01)$ but decreased to normal levels after the necessary treatment $(P<0.01)$.

Conclusion: The levels of HMGB1 mRNA and HMGB1 protein were elevated in patients with AOSC, which may play an important role in the inflammation of the bile duct and appears to be associated with the development of sepsis. This suggests the importance of monitoring HMGB1 levels in the management of AOSC-induced sepsis.

Keywords: AOSC, HMGB1, interleukin 10, TNF-alpha, sepsis, inflammation

Correspondence: Jianping Gong Chongqing Key Laboratory of Hepatobiliary Surgery and Department of Hepatobiliary Surgery, The Second Affiliated Hospital of Chongqing Medical University, No 76 Linjiang Road, Yuzhong District, Chongqing 400010 , People's Republic of China

Tel +86 I39 96286589

Fax +86236382 9191

Email gongjianpingII@I26.com

Chuanxin Wu

Chongqing Key Laboratory of Hepatobiliary Surgery and Department of Hepatobiliary Surgery, The Second Affiliated Hospital of Chongqing Medical University, No 76 Linjiang Road, Yuzhong District, Chongqing 40010,

People's Republic of China

Tel +8615923218833

Fax +86236382 9191

Email wu_chuanxin@live.com

\section{Introduction}

High-mobility group box 1 (HMGB1) is a chromatin-binding protein that is normally located predominantly inside the nucleus of cells. It is primarily responsible for transcription and growth factors. Recent studies identified HMGB1 as a mediator of inflammation when it is released into the extracellular environment, and it has been demonstrated to be a key factor for the development of acute inflammation. In response to infection, HMGB1 acts as an important proinflammatory cytokine that may lead to damage of cells and tissues. This process may progress to metabolic acidosis, multiorgan dysfunction syndrome, and sepsis. It is universally accepted that sepsis, which is fatal, is mediated by inflammatory mediators such as tumor necrosis factor alpha (TNF-alpha), interleukin 10 (IL-10), HMGB1, and so forth. All these factors have important actions in the startup, maintenance, and resolution of inflammation. TNF-alpha and IL-10 are early inflammatory factors that occur in the early phase of inflammation, during the onset of sepsis, and even a minimal 
delay in the therapeutic method directed against the early inflammatory factors make the management of this disease difficult for clinicians. Serum HMGB1 was significantly elevated from 8 to 72 hours after endotoxin exposure, in comparison with early inflammatory mediators TNF-alpha and interleukin. ${ }^{1,2}$ Treatment with delayed administration of anti-HMGB1 antibodies, ${ }^{3}$ a box of HMGB $1,{ }^{3}$ and ethyl pyruvate beginning as late as the disappearance of plasma TNF-alpha and interleukin significantly increases patient survival. ${ }^{4}$ Thus, targeting HMGB1 in the treatment of sepsis has a wider role than targeting early inflammatory cytokines such as TNF-alpha and IL-10 in the management of sepsis, and recent studies have reported several agents that target HMGB1 for the treatment of sepsis., 5

Cholangitis is the inflammation of biliary ducts, which usually begins extrahepatically and easily spreads intrahepatically, causing bacteremia. It was first described as fever, abdominal pain, and jaundice by Charcot in 1877 and was known as the "Charcot Triad." Later, in 1959, Reynolds added mental confusion and septic shock to the description, and it became known as "Reynolds Pentad." The obstruction of biliary ducts and the presence of superposing bacterial infection are common features in cholangitis. Obstruction of the biliary tract is primarily caused by choledocholithiasis. Moreover, malignancy, benign strictures, and interventions to the biliary duct may also be the cause of biliary obstruction. Acute obstruction of the biliary tract with the presence of pus is a severe form of cholangitis known as acute obstructive suppurative cholangitis (AOSC), which has very high mortality if the drainage is delayed. Charcot's Triad and Reynold's Pentad are difficult to use in clinical practice for the management of cholangitis. The recently released "TG13: Updated Tokyo Guidelines for the management of acute cholangitis and cholecystitis," published in 2013, have not been evaluated or revised. ${ }^{7}$ In our previous study, we found that nuclear factor $\mathrm{kB}$, a very early mediator of inflammation, was markedly increased in patients with severe acute cholangitis and that the degree of nuclear factor $\mathrm{kB}$ correlates with the degree of severity of the cholangitis. ${ }^{8}$ The degree of nuclear factor $\mathrm{kB}$ also correlates to clinical outcome. In another study of patients with cholangitis, we observed that TREM-1, which is also an early inflammatory cytokine, provided a useful early indicator for sepsis. ${ }^{9}$ However, little to no research exits regarding the late inflammatory mediators in the patients with AOSC or sepsis induced by AOSC. Recent studies have reported associations between HMGB1 and inflammation. ${ }^{10-12}$ Previously, we found that HMGB1 represents a potentially valuable marker for the diagnosis of acute appendicitis. ${ }^{13}$ However, the correlation between HMGB1 and AOSC pathogenesis is not yet fully understood. Our objective in the current study was to determine the role of HMGB1 in the inflammatory process of AOSC.

\section{Materials and methods}

All experimental protocols described in this study were approved by the research ethics review committee of the Second Affiliated Hospital of Chongqing Medical University and comply with the Chinese government guidelines. A total of 23 AOSC-induced patients with sepsis admitted to the Department of Hepatobiliary Surgery, the Second Affiliated Hospital of Chongqing Medical University, People's Republic of China, between November 2010 and June 2011 were randomly enrolled in the prospective study. During that period, 23 healthy individuals were also randomly selected from the Physical Examination Center of the Second Affiliated Hospital, Chongqing Medical University, People's Republic of China, to be used as a control group. Written consent was obtained from all volunteers. All AOSC-induced patients with sepsis and controls were between the ages of 18 and 80 years and were ethnically similar (Han Chinese). The selection of AOSC-induced patients with sepsis was based on diagnostic criteria and severity assessment of acute cholangitis, using the Tokyo Guidelines ${ }^{14}$ and International Sepsis Definitions Conference 2003. ${ }^{15}$ The patients involved in this study underwent biliary drainage via endoscopic retrograde cholangiopancreatography, or percutaneous transhepatic catheter drainage, or endoscopic ultrasound guided drainage, or open surgical drainage.

Peripheral blood samples of each AOSC-induced patient with sepsis were obtained predrainage, 12 hours postdrainage, and 24 hours postdrainage. In addition, peripheral blood samples from the individuals in the control group were obtained after overnight fasting.

Each sample was collected in $5 \mathrm{~mL}$ tubes, one with and one without heparin. The peripheral blood mononuclear cells (PBMCs) were immediately separated from the samples with heparin, using a lymphocyte separating medium and a density gradient centrifuge. After the blood clotted in the tube without heparin, the serum was separated and taken out of the tube and then stored at $-70^{\circ} \mathrm{C}$.

\section{mRNA measurement using real-time polymerase chain reaction}

PBMCs were isolated from the blood samples using a lymphocyte separation medium (Bosoter, People's Republic 
of China), a Ficoll density gradient isolating lymphocytes by centrifuging. The single-step technique of acid guanidinium thiocyanate chloroform extraction was used for total RNA extraction according to the instructions provided by the manufacturer. We determined the concentration of purified total RNA spectrophotometrically at a wavelength of $280 \mathrm{~nm}$. The HMGB1 mRNA and $\beta$-actin levels in blood samples were quantified in duplicate, using real-time polymerase chain reaction. We removed any potential DNA contamination using DNase I. We used $1 \mu \mathrm{g}$ total RNA from each sample for reverse transcription with an oligo (dT) and Super Script II reverse transcriptase to generate first-strand cDNA. Thermal cycling conditions were as follows: $2 \mathrm{~min}$ utes at $94^{\circ} \mathrm{C}$, followed by 35 cycles at $94^{\circ} \mathrm{C}$ for 30 seconds and at $60^{\circ} \mathrm{C}$ for 3 minutes on a Sequence Detection System (Applied Biosystems, Foster City, CA, USA). The expression was normalized to $\beta$-actin mRNA content. Sequences of the human primers for polymerase chain reaction were as follows: HMGB1 Bbox (127 bp): 5'-GCG GAC AAG GCC CGT TA-3' (sense), 5'-AGA GGA AGA AGG CCG AAG GA-3' (antisense); $\beta$-actin (255 bp): 5'-CAA AGA CCT GTA CGC CAA CA-3' (sense), 5'-GAA GCA TTT GCG GTG GAC-3' (antisense).

\section{HMGBI protein measurement using Western blot analysis}

PBMCs were isolated from the blood samples as previously described. The difference between HMGB1 protein expression levels was analyzed using a Western blot. Protein was extracted from PBMCs by homogenizing samples in a cell lysis buffer. Proteins were quantified using a Nanodrop 1000 (Thermo Scientific, Wilmington, DE, USA). Equal amounts of protein were loaded in each well for electrophoresis in $10 \%$ sodium dodecyl sulfate-polyacrylamide gels and then transferred to polyvinylidene fluoride micropoporous membranes (Millipore Corporation, Billerica, MA). The membrane containing the transferred proteins was blocked with $5 \%$ bovine serum albumin and then incubated with rabbit anti-HMGB1 polyclonal antibody (1:250; BD Pharmingen, San Diego CA), followed by an incubation with horseradish peroxidase-linked secondary antibody (1:1,000; Golden Bridge, Beijing, People's Republic of China). For standardization and comparison, the membranes were also hybridized to a primary anti $\beta$-actin antibody $(1: 1,000$; Santa Cruz Technology, Santa Cruz, CA, USA) or antihistine H 3.1 antibody (1:1,000; SAB, Pearland, TX, USA). The bands appearing on film were analyzed with Gene Tools software (Syngene, Frederick, MD, USA).

\section{Enzyme-linked immunosorbent assay}

The concentration of TNF-alpha and IL-10 in the serum was detected using commercially available enzyme-linked immunosorbent assay (ELISA) kits (R and D Systems, Minneapolis, $\mathrm{MN}, \mathrm{USA}$ ), following the manufacturer's instructions.

These three statistical analyses were completed using SPSS19.0 software for Windows (SPSS, Chicago, IL, USA). Data are presented as the mean \pm SEM unless otherwise indicated. A Kruskal-Wallis analysis was performed to ascertain that there is an overall difference, and a Mann-Whitney U test was used to test differences between the groups. $P$ values $<0.05$ were considered statically significant. $P$ values $<0.01$ were considered notably statistically significant.

\section{Results \\ Association of HMGBI mRNA expression in PBMCs of AOSC patients}

Total RNA was extracted from PBMCs, and the HMGB1 mRNA was amplified via real-time polymerase chain reaction to detect the expression of HMGB1 mRNA in PBMCs of patients with AOSC (Figure 1). As shown in Figure 3, the level of HMGB1 mRNA in PBMCs was $0.82 \pm 0.04$ in patients

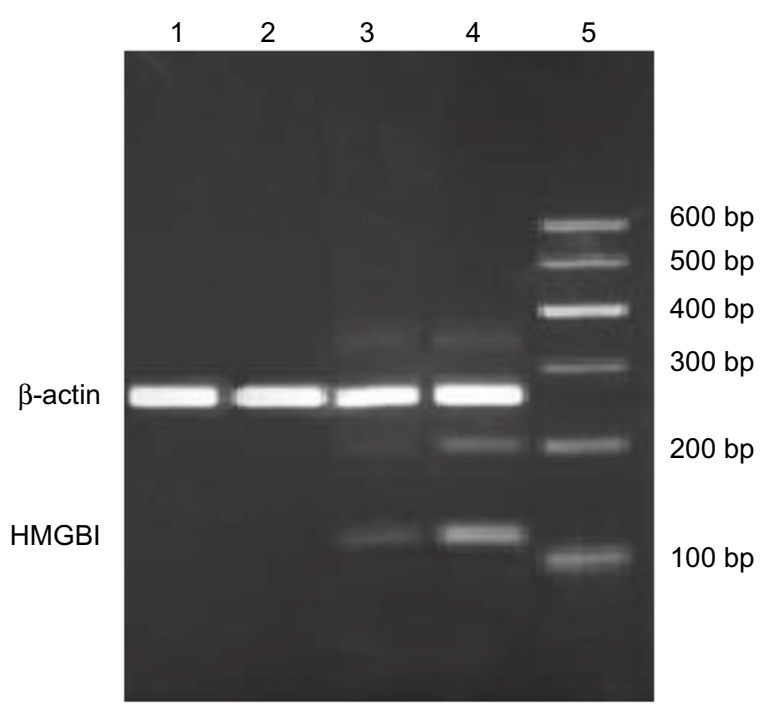

Figure I Expression of high-mobility group box I mRNA in peripheral blood mononuclear cells by real-time polymerase chain reaction analysis.

Notes: Total RNA was obtained from peripheral blood mononuclear cells of the control group and from acute obstructive suppurative cholangitis-induced sepsis patients (before the drainage, 12 hours after the drainage, and 24 hours after the drainage, respectively). Electrophoresis of high-mobility group box I gene fragment amplification product is shown. Lane I represents polymerase chain reaction product of control group, lane 2 represents polymerase chain reaction product of AOSCinduced sepsis group 24 hours after the drainage, lane 3 represents polymerase chain reaction product of AOSC-induced sepsis group 12 hours after the drainage, lane 4 represents polymerase chain reaction product of AOSC-induced sepsis group before the drainage and lane 5 represents DL2000 marker.

Abbreviations: AOSC, acute obstructive suppurative cholangitis; HMGBI, highmobility group box $\mathrm{I}$. 
with AOSC, which was significantly higher (62\%) than that of healthy donors $(0.31 \pm 0.06 ; P<0.01)$. We also found that the level of HMGB1 mRNA gradually decreased to $0.4 \pm 0.05$ and $0.38 \pm 0.07(P<0.01)$ after 12 and 24 hours postdrainage, respectively. The 12-hour postdrainage level of HMGB1 mRNA was decreased by $32 \%$ relative to the predrainage level.

\section{Association of HMGBI protein in PBMCs of patients with AOSC}

The HMGB1 protein levels in PBMCs of both groups, the patients with AOSC, and the healthy donors were tested using a Western blot analysis (Figure 2). As shown in Figure 3, we found that the level of the HMGB1 protein in patients with AOSC was $0.81 \pm 0.03$, which was significant and $57 \%$ higher than that of $0.35 \pm 0.05(P<0.01)$ seen in healthy donors. The level of HMGB1 proteins gradually decreased to $0.56 \pm 0.05$ and $0.42 \pm 0.07(P<0.01)$ after 12 and 24 hours postdrainage, respectively. The 12-hour postdrainage level of HMGB1 protein decreased by $37 \%$ compared with the predrainage level.

\section{Association of TNF-alpha and IL- 10 expression in the serum of patients with AOSC}

The level of TN-alpha and IL-10 proteins in the serum of each sample from patients with AOSC and healthy donors was determined using commercially available ELISA kits. We found that TNF-alpha and IL-10 were significantly higher in the patients than in healthy donors and that they gradually decreased after biliary passage drainage (Figure 4). The average predrainage value of TNF-alpha was $469 \pm 35 \mathrm{pg} / \mathrm{mL}$, which decreased to $243 \pm 26 \mathrm{pg} / \mathrm{mL}$ and $132 \pm 47 \mathrm{pg} / \mathrm{mL}$ after 12 and 24 hours postdrainage, respectively; it was $102 \pm 16$ $\mathrm{pg} / \mathrm{mL}$ in the healthy controls. In the same regard, the average

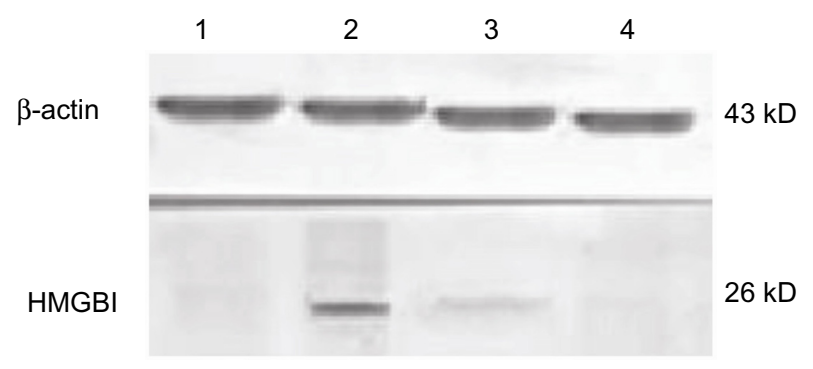

Figure 2 Western blotting analysis of high-mobility group box I protein expression in peripheral blood mononuclear cells.

Notes: Western blotting was performed to detect protein level of high-mobility group box I. Total protein was extracted from peripheral blood mononuclear cells. Lane I represents the control, lane 2 represents preoperation, lane 3 represents postoperation 12 hours, and lane 4 represents postoperation 24 hours. Abbreviation: HMGBI, high-mobility group box $\mathrm{I}$.

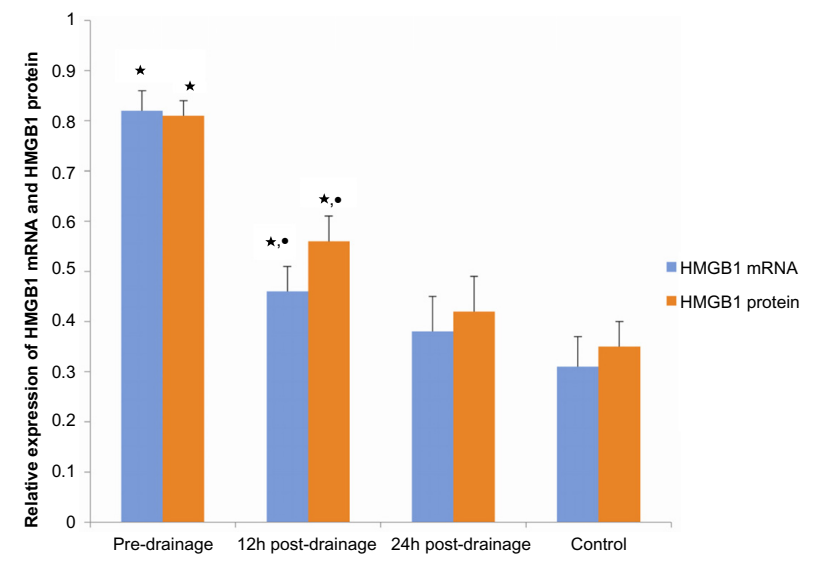

Figure 3 Comparison of high-mobility group box I (HMGBI) mRNA and highmobility group box I protein expression among patients with acute obstructive suppurative cholangitis and controls $(n=23)$.

Notes: ${ }^{\star}$ Compared with control, $P<0.01$; ${ }^{\circ}$ compared with predrainage, $P<0.01$.

predrainage value of IL-10 was $33.369 \pm 6.443 \mathrm{pg} / \mathrm{mL}$, which decreased to $20.562 \pm 5.630 \mathrm{pg} / \mathrm{mL}$ and $13.742 \pm 3.374 \mathrm{pg} /$ $\mathrm{mL}$ after 12 and 24 hours postdrainage, respectively; it was $11.490 \pm 1.088 \mathrm{pg} / \mathrm{mL}$ in healthy controls (Figure 5).

\section{Discussion}

Acute cholangitis is a systemic infectious disease characterized by acute inflammation and biliary tract infection. Acute cholangitis occurs as a result of bacterial colonization within the biliary tract, which increases the internal pressure of the biliary tract and causes a backflow of bacteria and endotoxins within the circulatory system. ${ }^{16,17}$ AOSC is a severe form of cholangitis in which pus collects in the biliary tract. Drainage of the biliary tract should be performed as soon as possible in patients with AOSC; otherwise, translocation of

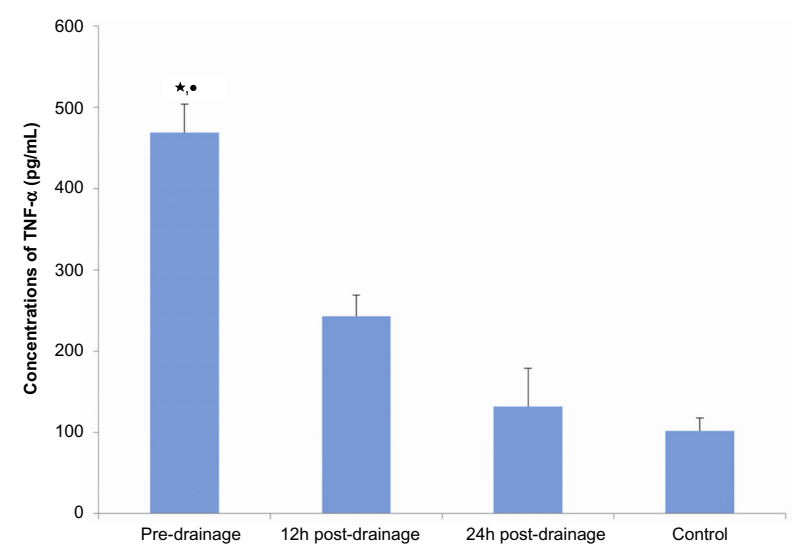

Figure 4 The analysis of tumor necrosis factor alpha by enzyme-linked immunosorbent assay (ELISA) in serum of operated and healthy controls.

Notes: Tumor necrosis factor alpha (TNF- $\alpha$ ) was quantitated using an ELISA kit. Data are expressed as median for each sample. Experiments were performed at least three times, getting similar results. ${ }^{\star}$ Compared with control, $P<0.01$; ${ }^{\circ}$ compared with 12 hours postdrainage, $P<0.01$. 


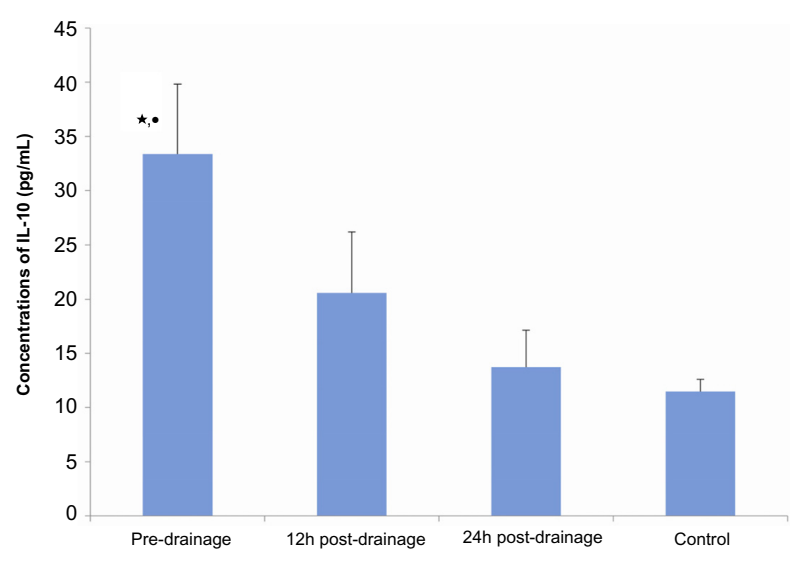

Figure 5 The analysis of interleukin 10 (IL-I0) by enzyme-linked immunosorbent assay (ELISA) in serum of operated and healthy controls.

Notes: Tumor necrosis factor alpha was quantitated using ELISA kit. Data are expressed as median for each sample. Experiments were performed at least three times getting similar results. ${ }^{\star}$ Compared with control, $P<0.01$; 'compared with 12 hours postdrainage, $P<0.01$.

bacteria into the bloodstream results in septicemia, an often fatal complication of acute cholangitis that induces severe organ damage and high mortality. There was no appropriate guideline for the diagnosis and management of acute cholangitis before the TG13: Updated Tokyo Guidelines for the management of acute cholangitis and cholecystitis. The symptoms and signs described as Charcot's Triad and Reynold's Pentad are difficult for physicians to use in diagnostic and management strategies in clinical settings. The newly published TG13 is considered to have better diagnostic and management capacity. However, TG13 lacks sufficient feedback and revisions. Within 1 year, several studies found that the diagnostic criteria of TG13 fail to identify severe cases of cholangitis that require emergency biliary drainage. ${ }^{18}$ Hence, the management strategy of TG13 may not be especially specific to AOSC, which is a severe form of cholangitis in which emergency biliary drainage is a necessity. Further study of the diagnosis and management of AOSC is necessary. A marker to be used to diagnose AOSC-induced cholangitis would present a helpful step in the management of AOSC. Recently, several studies have found that HMGB1 is an important agent for the diagnosis and management of sepsis. The present study focused on the correlation of HMGB1 with cholangitis in patients with AOSC.

Many clinical and experimental studies have described the release of HMGB1 during inflammation. ${ }^{13,19}$ It has been shown that HMGB1 is a mediator of inflammation when it is released extracellularly. The extracellular release of HMGB1 may occur either through active release by immune cells or passive secretion after cell death. Inside the nucleus, HMGB1 functions as a protein for binding chromatin and helps in the transcription process of genes. ${ }^{20}$ Extracellular release of HMGB1 is a result of translocation of HMGB1 from the nucleus to the cytoplasm, which involves acetylation, ${ }^{21}$ phosphorylation, ${ }^{22}$ and methylation. ${ }^{23}$ Extracellular HMGB1 then exerts biological expression via its receptors. HMGB1 signaling is mediated by several receptors, including Receptor for Advanced Glycation Endproduct and Toll-like receptors. ${ }^{24,25}$ During infection, endotoxins are produced by bacteria that activate monocytes and macrophages such as TNF-alpha, IL-10, and so on. These cytokines promote HMGB1 translocation from the nucleus into the cytoplasm and further enter into transcription processes by themselves. ${ }^{5,26,27}$ These produced HMGB1 proteins, act as cytokines, which further induce the stimulation of other inflammatory agents, resulting in a worsening in the severity of inflammation.

Our study demonstrated that the expression levels of TNF-alpha, IL-10, and HMGB1 were significantly higher in patients with AOSC than in normal controls. After drainage, the level of HMGB1, TNF-alpha, and IL-10 in the patients with AOSC decreased as infection gradually decreased, indicating that with the exacerbation of AOSC, HMGB1 is positively correlated with the disease. Increased expression ofTNF-alpha and IL-10 has also been observed in noninflammatory diseases, whereas increased expression of HMGB1 is specifically observed in the PBMCs and serum in patients with sepsis. Recent studies have also reported that the severity of the disease increases with the increase in the expression of HMGB1. In severely burned patients, it has been reported that the level of HMGB1 expression is significantly higher in deceased patients than in survivors. ${ }^{28}$ HMGB1 has been studied for more than 4 decades, and in recent years, it has gained attention as a potential marker of sepsis. Our previous study demonstrated that HMGB1 expression may represent a novel marker for the diagnosis of acute appendicitis and also aids physicians in deciding the severity of the disease. ${ }^{13}$ Because TNF-alpha and IL-10 are early markers of inflammation, whereas expression of HMGB1 is found in late stages of sepsis, HMGB1's significance in patients with sepsis can be higher than early inflammatory factors such as TNF-alpha and IL-10. At this time, several animal and clinical studies have also demonstrated a protective effect of anti-HMGB1 agents on sepsis. It should be noted that if sepsis is a result of HMGB1, there will be an adequate time interval to detect it and administer anti-HMGB1 for the therapeutic management of sepsis.

In patients with AOSC-induced sepsis, bacteria can easily enter the circulatory system because there is increased 
intraductal pressure caused by blockage of the biliary tract. These bacteria produce endotoxins and proinflammatory agents. The produced proinflammatory agents activate monocytes and macrophages, which cause the release of HMGB1 in the extracellular environment. The released HMGB1 acts as a proinflammatory factor that worsens the inflammation and affects distal organs. ${ }^{26,27}$ From this point of view, the increased level of HMGB1 could be directly correlated with the severity of ASOC-induced sepsis and, hence, can be useful as a diagnostic marker in patients with AOSC. In addition, controlling the expression of HMGB1 with an anti-HMGB1 agent may improve the efficacy of AOSC therapy by managing systemic inflammation and reducing organ injury by sepsis. However, there is a lack of clinical evidence of suppressing increased expression of HMGB1 in patients with sepsis, which requires further clarification by clinical trials results.

\section{Conclusion}

Our study demonstrated the increased expression of HMGB1 in peripheral blood mononuclear cells of AOSC-induced patients with sepsis, which may play an important role for the progression of sepsis with the patients with AOSC. The sample size of our study is small, and we did not include inflammation caused by other disorders that represent important parameters that affect sensitivity and specificity of HMBG1 in patients with AOSC. The mechanism of HMGB1 in patients with AOSC remains unclear and requires further study. This result is not designed to control AOSC-induced sepsis through anti-HMGB1 therapy, and this needs to be further researched. Although we have yet to complete further research to demonstrate the sensitivity and specificity of HMGB1 in the diagnosis and therapy for patients with AOSC, combined with other diagnostic markers, HMGB1 could provide a useful biomarker for AOSC-induced sepsis.

\section{Acknowledgments}

This study was supported by grants from the National Natural Science Foundation of China (No 81401622 and No 81071339).

\section{Disclosure}

The authors report no conflicts of interest in this work.

\section{References}

1. Wang H, Bloom O, Zhang M, et al. HMG-1 as a late mediator of endotoxin lethality in mice. Science. 1999;285(5425):248-251.
2. Yang H, Ochani M, Li J, et al. Reversing established sepsis with antagonists of endogenous high-mobility group box 1. Proc Natl Acad Sci U S A. 2004;101(1):296-301.

3. Li X, Wang LK, Wang LW, Han XQ, Yang F, Gong ZJ. Blockade of high-mobility group box-1 ameliorates acute on chronic liver failure in rats. Inflammation Research: Official Journal of the European Histamine Research Society ... [et al.]. Jul 2013;62(7):703-709.

4. Ulloa L, Ochani M, Yang H, et al. Ethyl pyruvate prevents lethality in mice with established lethal sepsis and systemic inflammation. Proc Natl Acad Sci U SA. 2002;99(19):12351-12356.

5. Fink MP. Neuropeptide modulators of high mobility group box 1 secretion as potential therapeutic agents for severe sepsis. Am J Pathol. 2008;172(5):1171-1173.

6. Yoo H, Ku SK, Baek YD, Bae JS. Anti-inflammatory effects of rutin on HMGB1-induced inflammatory responses in vitro and in vivo. Inflammation Research: Official Journal of the European Histamine Research Society ... [et al.]. Mar 2014;63(3):197-206.

7. Takada T, Strasberg SM, Solomkin JS, et al. TG13: Updated Tokyo Guidelines for the management of acute cholangitis and cholecystitis. Journal of Hepato-Biliary-Pancreatic Sciences. Jan 2013;20(1):1-7.

8. Gong JP, Liu CA, Wu CX, Li SW, Shi YJ, Li XH. Nuclear factor kB activity in patients with acute severe cholangitis. World J Gastroenterol. 2002;8(2):346-349.

9. Liao R, Liu Z, Wei S, Xu F, Chen Z, Gong J. Triggering receptor in myeloid cells (TREM-1) specific expression in peripheral blood mononuclear cells of sepsis patients with acute cholangitis. Inflammation. 2009;32(3):182-190.

10. El Gazzar M. HMGB1 modulates inflammatory responses in LPSactivated macrophages. Inflammation Research: Official Journal of the European Histamine Research Society ... [et al.]. Apr 2007;56(4): $162-167$.

11. Kao RL, Xu X, Xenocostas A, et al. Induction of acute lung inflammation in mice with hemorrhagic shock and resuscitation: role of HMGB1. J Inflamm (Lond). 2014;11(1):30.

12. Hou C, Kong J, Liang Y, et al. HMGB1 contributes to allergen-induced airway remodeling in a murine model of chronic asthma by modulating airway inflammation and activating lung fibroblasts. Cell Mol Immunol. 2014;(Aug):25.

13. Wu C, Sun H, Wang H, et al. Evaluation of high mobility group box 1 protein as a presurgical diagnostic marker reflecting the severity of acute appendicitis. Scand J Trauma Resusc Emerg Med. 2012; 20(1):61.

14. Wada K, Takada T, Kawarada Y, et al. Diagnostic criteria and severity assessment of acute cholangitis: tokyo Guidelines. $J$ Hepatobiliary Pancreat Surg. 2007;14(1):52-58.

15. Levy MM, Fink MP, Marshall JC, et al; SCCM/ESICM/ACCP/ATS/ SIS. $2001 \mathrm{SCCM} / \mathrm{ESICM} / \mathrm{ACCP} / \mathrm{ATS} /$ SIS International Sepsis Definitions Conference. Crit Care Med. 2003;31(4):1250-1256.

16. Kimura Y, Takada T, Strasberg SM, et al. TG13 current terminology, etiology, and epidemiology of acute cholangitis and cholecystitis. Journal of Hepato-Biliary-Pancreatic Sciences. Jan 2013;20(1):8-23.

17. Mosler P. Diagnosis and management of acute cholangitis. Curr Gastroenterol Rep. 2011;13(2):166-172.

18. Shinya S, Sasaki T, Yamashita Y, et al. Procalcitonin as a useful biomarker for determining the need to perform emergency biliary drainage in cases of acute cholangitis. Journal of Hepato-Biliary-Pancreatic Sciences. Oct 2014;21(10):777-785.

19. Wu CX, Sun H, Liu Q, Guo H, Gong JP. LPS induces HMGB1 relocation and release by activating the NF- $\mathrm{KB}-\mathrm{CBP}$ signal transduction pathway in the murine macrophage-like cell line RAW264.7. J Surg Res. 2012;175(1):88-100.

20. Stott K, Tang GS, Lee KB, Thomas JO. Structure of a complex of tandem HMG boxes and DNA. J Mol Biol. 2006;360(1):90-104.

21. LiY, Gan CP, Zhang S, et al. FIP200 is involved in murine pseudomonas infection by regulating HMGB1 intracellular translocation. Cell Physiol Biochem. 20133(6):1733-1744. 
22. Ge X, Antoine DJ, Lu Y, et al. High mobility group box-1 (HMGB1) participates in the pathogenesis of alcoholic liver disease (ALD). J Biol Chem. 2014;289(33):22672-22691.

23. Wu F, Zhao ZH, Ding ST, Wu HH, Lu JJ. High mobility group box 1 protein is methylated and transported to cytoplasm in clear cell renal cell carcinoma. Asian Pac J Cancer Prev. 2013;14(10):5789-5795.

24. Park JS, Gamboni-Robertson F, He Q, et al. High mobility group box 1 protein interacts with multiple Toll-like receptors. Am J Physiol Cell Physiol. 2006;290(3):C917-C924.

25. Ivanov S, Dragoi AM, Wang X, et al. A novel role for HMGB1 in TLR9-mediated inflammatory responses to CpG-DNA. Blood. 2007;110(6):1970-1981.
26. Pisetsky DS. The expression of HMGB1 on microparticles released during cell activation and cell death in vitro and in vivo. $\mathrm{Mol} \mathrm{Med}$. 2014;20(1):158-163.

27. Rendon-Mitchell B, Ochani M, Li J, et al. IFN-gamma induces high mobility group box 1 protein release partly through a TNF-dependent mechanism. J Immunol. 2003;170(7):3890-3897.

28. Huang LF, Yao YM, Dong N, Yu Y, He LX, Sheng ZY. Association of high mobility group box-1 protein levels with sepsis and outcome of severely burned patients. Cytokine. 2011;53(1):29-34.

Journal of Inflammation Research

\section{Publish your work in this journal}

The Journal of Inflammation Research is an international, peer-reviewed open-access journal that welcomes laboratory and clinical findings on the molecular basis, cell biology and pharmacology of inflammation including original research, reviews, symposium reports, hypothesis formation and commentaries on: acute/chronic inflammation; mediators of inflamma-

\section{Dovepress}

tion; cellular processes; molecular mechanisms; pharmacology and novel anti-inflammatory drugs; clinical conditions involving inflammation. The manuscript management system is completely online and includes a very quick and fair peer-review system. Visit http://www.dovepress.com/ testimonials.php to read real quotes from published authors.

\footnotetext{
Submit your manuscript here: http://www.dovepress.com/journal-of-inflammation-research-journal
} 